\title{
Sagesse et barbarie en terre gauloise. La violence fondatrice dans la bande dessinée Astérix ${ }^{1}$
}

\author{
Jean-Pierre Thomas \\ Collège universitaire Glendon (Université York)
}

Contre la force, utilisons la force! Souviens-toi de nos campagnes, César! Nous avons fait plier le monde devant nos légionnaires!

Obélix et compagnie, p. 13.

\footnotetext{
1 Je tiens à transmettre mes sincères remerciements à Sylvain Rheault qui, lors de la « Fête de la B.D. », qui a eu lieu à l'Université de Régina en mai 2009, m'a permis, par ses propos éclairés, d'affiner ma réflexion sur quelques-unes des questions traitées ici.
} 
Jeunes et moins jeunes connaissent bien le scénario, qui se répète, à quelques variantes près, d'album en album : acculés par les Romains à une résistance apparemment illusoire l'ennemi les domine à 100 contre un -, terrés dans un village entouré d'avant-postes garnis de soldats arrivistes, un groupe de Gaulois armés d'une potion qui les rend «surhumain[s] [et] irréductibles ${ }^{2}$ » renversent l'adversité, à leur plus grand plaisir. Altercations et combats se multiplient entre les belligérants et les rebelles aplatissent leurs adversaires jour après jour, se prévalant d'une violence dont on peut se demander si elle relève de la nécessité ou si elle n'apparaît pas plutôt çà et là gratuite. René Goscinny et Albert Uderzo, les créateurs de la bande dessinée Astérix, auraient-ils mis au point un ingénieux stratagème leur permettant de distiller les effets d'une violence cathartique auprès de générations de lecteurs? La potion concoctée par le druide Panoramix, qui métamorphose les héros gaulois en des simulacres de superhéros l'espace de quelques heures, semble faire simultanément d'eux des êtres violents et incontrôlables - témoin cet Obélix qui, véritable «incarn[ation de] la bête sauvage agressive» selon Bernard Lassablière (47), frappe Romains et pirates sans se formaliser des conséquences. Les Gaulois représenteraient-ils l'incarnation parfaite de la barbarie?

Il s'agira ici d'évaluer les enjeux fonctionnels de la violence dans la série Astérix et de déterminer, au-delà des multiples représentations caricaturales données du fait violent, si cette dernière conduit à une frénésie aveugle ou si, au contraire, elle ne joue pas plutôt un rôle quelque peu salutaire. Le caractère parodique de la bande dessinée Astérix relativise-t-

2 Voir la page 3 de chaque album de la série Astérix. 
il la fonction attribuée au fait violent au point de transformer celui-ci en une simple donnée secondaire dans un cadre plus large, la caricature socioculturelle ? La fête débridée à laquelle convie tout album de la série, point culminant d'une débâcle de combats, marque-t-elle le Grand CEuvre de Goscinny et d'Uderzo du sceau d'une apologie de la violence, enlevant à cette dernière son caractère de légitime défense, ou la littérature ne se dote-t-elle pas, avec les aventures d'Astérix, d'un moyen novateur et bénéfique de contourner les interdits? Je me livrerai dans un premier temps à l'établissement d'une nomenclature des différents visages sous lesquels est représentée la violence dans la série, histoire de marquer les limites du propos. J'étudierai par la suite les fonctions remplies par la violence dans certains albums, pour finalement tenter quelques explications d'ordre anthropologique. Bien que j'aie consulté les vingt-quatre premiers albums de la série (ceux qui ont été scénarisés par René Goscinny) pour me livrer à ce travail, je concentrerai la réflexion sur quelques titres précis, indiqués au fur et à mesure de la progression.

\section{Appel(s) à la violence : quelques constats utiles}

Si nous acceptons la définition courante du phénomène de violence, celle d'«un rapport de puissance entre les hommes qui renonce aux autres méthodes possibles d'entretenir des relations entre les êtres et qui essaie de forcer, directement ou indirectement, les individus ou les groupes d'agir contre leur volonté ou d'exécuter les desseins d'une volonté qui leur est étrangère » (Freund, p. 35), force est de constater que la bande dessinée Astérix s'avère d'une richesse quasi illimitée, ce que le paratexte révèle à travers un réseau sémantique constitué de 
marques précises. Avec des titres comme Le Combat des chefs, La Zizanie ou Astérix gladiateur, le lecteur est mis sur la piste. Les illustrations des pages de couverture renchérissent, la rixe (qu'elle soit le fait d'individus ou de groupes ${ }^{3}$ ) y jouant un rôle capital. Quant à la double page de garde placée au début et à la fin de chaque album de la série, y est présenté, en addition d'une nouvelle démonstration de force sans équivoque, un paradoxe qui accompagnera les personnages dans leurs aventures: les manifestations de violence semblent relever de la pure formalité pour les Gaulois, ce que prouve la posture d'Obélix qui, bien que se prétendant affaibli, n'en coince pas moins sans difficulté trois Romains sous son bras. Cette double page indique que la violence est à considérer dans des optiques variées: l'attitude conquérante, diaïrétique manifestée par la verticalité de la distribution des baffes dans la page de gauche, est " euphémisée », à travers le processus de lecture qui conduit l'œil vers la page de droite, par la rondeur du ventre d'Obélix, de la marmite ainsi que de l'éventail formé par les corps des soldats romains. La verticalité de la vapeur qui émane de la marmite se trouve pour sa part amenuisée par sa forme en zigzag. Cette lecture, pour l'instant sommaire, permet de constater, à la suite de Lassablière, que «la violence des affrontements ne fait aucun doute » (p. 77), bien qu'elle donne l'impression de ne pas pouvoir être considérée de manière unilatérale.

Cette prise de contact initiale avec l'attitude agressive des personnages principaux de la série se voit rapidement confirmée par le contenu des albums. Après la lecture de

\footnotetext{
3 Voir, par exemple, les pages de couverture des albums Astérix le Gaulois, Astérix gladiateur ou Le Cadeau de César.
} 
quelques récits, un personnage type se repère, sorte de matrice d'après laquelle la plupart des villageois paraissent avoir été façonnés : les Gaulois sont «irréductibles, courageux, teigneux, têtus, ripailleurs, bagarreurs et rigolards » (Goscinny et Uderzo, 1966, p. 5). Toujours prompts à se lancer dans la mêlée, comme l'affirme leur chef Abraracourcix dans l'album Le devin ${ }^{4}$, ces guerriers colériques et impatients sont marqués par un attribut singulier, leur force, qui, décuplée par une rasade de potion magique, atteint une envergure surhumaine et transforme chacun en l'équivalent d'une armée. À la question «Ça une bande décurion? Mais ils ne sont que deux!», posée dans Astérix et les Goths par un soldat romain, ce dernier répond luimême après une courte altercation: « Tu avais raison décurion... C'était une bande!» (Goscinny et Uderzo, 1963, p. 15) Cet attribut sous-tend à vrai dire la représentation offerte par Goscinny et Uderzo du comportement de leurs personnages et du tempérament querelleur de ceux-ci, ce qui entraîne Panoramix, dans Obélix et compagnie, à les définir comme relevant de "coutumes ancestrales » et Astérix, de «bon vieux train-train quotidien » (Goscinny et Uderzo, 1976, p. 46). Voilà une admission directe de la nécessité de pratiquer la violence : la force a une fonction précise dans cet univers. Le conflit caractérisera tous les albums de la série, au point où l'atmosphère normale du village gaulois est bien celle de l'altercation. Les Gaulois ne reçoivent-ils pas d'ailleurs la protection d'un dieu celte associé à la guerre, Toutatis, par le

\footnotetext{
${ }^{4}$ Abraracourcix donne l'explication suivante au mage : « Excuse mes hommes, devin. Ils passent leur temps à se bagarrer ». Son interlocuteur de répondre : " Je sais », comme s'il allait de soi que ce peuple belliqueux se complaît dans la bagarre (Goscinny et Uderzo, 1972b, p. 8)
} 
nom duquel ils jurent sans discontinuer ${ }^{5}$ ? Normal que, par extension, ils se sentent tenus de guerroyer à leur tour. Les Gaulois sont donc friands de bataille. En fait, " pourvu qu'il y ait des sangliers et des Romains...» (Goscinny et Uderzo, 1974, p. 21), selon les dires du barde Assurancetourix, tout leur est égal. Quand il n'est pas possible d'affronter des adversaires dignes de ce nom, les Gaulois se tournent vers l'intérieur des murs de leur village et se livrent à des combats contre leurs pairs - le running-gag de la violence perpétrée contre le barde est à ce titre bien connu. Même que, pour ce petit peuple singulier, le calme est synonyme de bataille, ce que montre la toute première case de l'album Astérix chez les Belges : "Sous un beau ciel ensoleillé, c'est une journée placide, semblable à toutes les autres, qui se déroule dans le paisible petit village gaulois...» (Goscinny et Uderzo, 1979, p. 5), relate le narrateur pendant qu'une bagarre impliquant la quasi-totalité des habitants du village se produit sous les yeux du lecteur.

Il est possible de repérer deux types de violence principaux représentés dans les albums. J'appellerai le premier type "violence instituée ", pour utiliser une terminologie chère à l'anthropologue Michel Maffesoli (1984, p. 8). Il s'agit d'une violence servant de moyen pour réaliser quelque objectif. Les Gaulois apparaissent, dès le premier album, coincés dans une position de résistance à l'oppresseur romain. Dans Astérix ou la parodie des identités, Nicolas Rouvière écrit: "La bande dessinée [Astérix] promeut [...] le droit légitime qu'a toute

\footnotetext{
5 Olivier Andrieu a relevé un total de 120 appels à Toutatis dans l'ensemble des albums (y compris ceux qu'Uderzo a scénarisés). Bien que d'autres divinités soient invoquées dans la série, le dieu de la guerre remporte la palme sans difficulté : Bélénos vient en second avec seulement trente-trois appels, tandis que Belisama en reçoit neuf (Andrieu, p. lxvi).
} 
culture de vivre librement son développement propre, sans subir l'oppression militaire d'une puissance impérialiste " (2008, p. 291). Voilà pourquoi les Gaulois sont toujours sur le qui-vive, prêts à engager le combat, toutes les raisons leur paraissant bonnes pour repousser le despotisme romain et même, à l'occasion, faire ombrage à celui-ci. La légitime défense semble caractériser leur attitude à l'égard de l'Empire. Un coup d'œil sur l'incipit du premier album de la série renseigne à ce propos (Goscinny et Uderzo, 1961, p.5). La case initiale de la première page présente les Romains sur le champ de bataille, engagés dans un mouvement visant à repousser les Gaulois vers la gauche, position de faiblesse, à tel point que ceux-ci sont situés hors cadre. Quelques artefacts gaulois brisés ainsi que le faciès déterminé des soldats rendent les prémisses claires : les Romains veulent soumettre les Gaulois. Dans la deuxième case, le chef Vercingétorix dépose les armes aux pieds de César (toujours dans un mouvement orienté vers la gauche, tandis que l'empereur est tourné vers la droite, position de force), non sans toutefois user de violence dans la défaite, promesse d'une éventuelle récidive de la part du peuple gaulois. La deuxième bande rend écrasante la domination romaine, laquelle s'étend au peuple germain et à l'ensemble de la Gaule, dont la Terre Mère craquelle sous la pression de l'aigle romaine apposée en son centre. Seul résiste un petit village marqué par la rondeur euphémisante de ses huttes et de la loupe qui en agrandit les contours, épine dans le pied d'un César qui, dans la case suivante, ne peut plus regarder vers la droite, n'étant plus le maître incontesté de son royaume. Astérix surgit dans ce contexte et, instantanément, une troupe romaine le menace, brimant sa liberté et cherchant à s'approprier la position de force (lances et regards pointent vers la droite). La dernière 
bande, par l'utilisation d'une représentation imagée de la force et d'onomatopées révélatrices des ravages causés par le héros, renverse la situation : désormais, les vaincus peuvent prétendre à la résistance, et la dernière case, avec un mouvement surdéterminé vers la gauche (par le déplacement d'Astérix, les pointes des lances brisées, le geste d'un Romain, un arbre qui tangue), rend compte du caractère définitif de la scène. Ayant été menacé de violence, Astérix réplique au nom du peuple gaulois par une violence légitimée. Résultat : les Romains font à leur tour l'expérience du chaos et y perdent leur latin, comme le mentionne l'instance narrative, signe précurseur du désordre à venir. Placée sous le signe de la violence, la série progressera tout en heurts.

L'autre type de violence, plus fréquent, sera nommé, d'après les termes de Maffesoli, «violence fondatrice ou instituante». Cette violence prend plusieurs couleurs et concerne le plus souvent les sens: musicale ${ }^{6}$, simplement sonore $^{7}$, olfactive ${ }^{8}$, gustative $^{9}$ ou évidemment physique, elle semble motivée par le désir de générer du plaisir. Fin en soi et non moyen, elle témoigne du tempérament festif des Gaulois, qui font par exemple se prolonger les combats à outrance : Obélix se plaint constamment $\mathrm{du}$ fait que les batailles se terminent trop tôt et, déjà dans le premier album, il souhaite

6 Le barde fait fuir êtres humains et animaux chaque fois qu'il utilise ses instruments, comme c'est le cas dans Astérix gladiateur (Goscinny et Uderzo, 1964, p. 6).

7 Le chahut causé par Astérix et Obélix dans Les Lauriers de César réveille toute une maisonnée romaine (Goscinny et Uderzo, 1972a, p. 25).

8 Dans Le Devin, Panoramix prépare une potion destinée, par l'odeur qu'elle dégage, à repou, sser une armée romaine (Goscinny et Uderzo, 1972b, p. 30).

${ }^{9}$ Cette fois, c'est Astérix qui concocte un repas odieux pour ses hôtes dans Les Lauriers de César (Goscinny et Uderzo, 1972a, p. 21). 
« ranim[er les victimes] pour recommencer» (Goscinny et Uderzo, 1961, p. 13). C'est que la spécialité culturelle de ce petit groupe est, comme le précise Astérix en envoyant valdinguer un ennemi, " [1]a châtaigne !» (Goscinny et Uderzo, 1965a, p. 48) Bons vivants, les Gaulois cherchent à s'amuser, et la rixe compte pour beaucoup dans ce processus, avec comme conséquences que les manifestations d'animosité prennent çà et là une teneur gratuite, ce qu'il serait facile de reprocher aux auteurs, d'autant plus que cette violence touche, par l'entremise du langage, le quotidien de l'individu ainsi que le processus de structuration de sa pensée. De fait, la violence physique cède souvent le pas devant une violence verbale qui, elle aussi diversifiée, accentue le phénomène d'agression. Jurons, insultes et onomatopées incisives (dans la mesure où elles reproduisent le bruit de coups assenés - voir, par exemple, Goscinny et Uderzo, 1979, p. 42) pullulent dans la bande dessinée. En outre, l'utilisation de sigles hiéroglyphiques (Goscinny et Uderzo, 1962, p. 5) et, d'une manière récurrente et prononcée (au point où il en est fait usage presque à chaque page), de caractères gras (par exemple, Goscinny et Uderzo, 1975, p. 44) qui peuvent disputer l'espace réservé au dessin ou empiéter sur les cases avoisinantes (Goscinny et Uderzo, 1969, p. 20), rend les vignettes provocantes et peut-être un tantinet agaçantes à la longue. Quant à l'insulte "Ils sont fous ces... [et ajouter un gentilé] », utilisée à répétition et agrémentée d'un coup de doigt sur la tempe, elle relève aussi de la violence verbale, car elle figure une attaque contre ce qu'a de plus précieux celui qui en est le destinataire : son identité.

La bataille rythme donc l'ensemble de la vie des Gaulois et, malgré sa dimension caricaturale évidente, la bande dessinée n'est pas loin de confiner ici et là à un éloge de la 
violence. Telle est du moins la représentation que le lecteur décode au fil de sa progression : maîtres de leur art, Goscinny et Uderzo ont mis en œuvre de nombreuses stratégies narratives afin de nantir la représentation qu'ils donnent de la violence d'une teneur iridescente. Dans La Grande Traversée, comment les autochtones immortalisent-ils la visite d'Astérix et d'Obélix en Amérique sinon par la transcription graphique de leurs exploits? (Goscinny et Uderzo, 1975, p. 30) Il semble que pratiquement toute action implique un acte violent de quelque type pour les Gaulois, même les marques de gentillesse et les excuses présentées dans Astérix légionnaire (Goscinny et Uderzo, 1967 b, p. 17 et 25). La violence permet de régler tout conflit, si bien que, banalisée, elle finit par relever de la pure formalité. Rien de plus normal pour les héros que d'entretenir une conversation pendant qu'ils se bagarrent dans La Serpe d'or (Goscinny et Uderzo, 1962, p. 8). La perspective de devoir se lancer dans la bataille n'angoisse jamais les Gaulois et une démonstration de violence devient vite le dénouement attendu de l'intrigue, d'où caricature. Le point culminant de ce phénomène réside dans le caractère elliptique que prend la rixe dans les albums. Les combats sont si prévisibles et si communs qu'afin d'en éviter la reprise ou pour euphémiser la dimension exagérément violente de la série, ils sont souvent passés sous silence : à la page 20 du Bouclier arverne, les compagnons vont affronter une troupe de Romains et ils en reviennent sans que rien ait été montré du combat, sauf son résultat. Même procédé dans Le Devin, où un bateau pirate est coulé hors cadre (Goscinny et Uderzo, 1972b, p. 38). Il arrive aussi que l'action soit simplement commentée par des observateurs durant son déroulement, sans que le lecteur en voie quoi que ce soit (Goscinny et Uderzo, 1973, p.43). Goscinny et Uderzo ont 
choisi, afin de limiter l'impact d'une violence déjà passablement présente dans les albums, de la dissimuler de temps à autre, d'en restreindre quelque peu les effets en la passant sous silence.

Si les Gaulois sont contraints de recourir à la force, ne devrait-on pas en mettre le blâme sur les Romains, lesquels se targuent de représenter le sommet de la civilisation mais n'en font pas moins preuve d'un goût prononcé pour la guerre? Les plans de conquête de César sont pour le moins agressifs dans ce qu'ils ont de définitif: l'empereur ne veut ni plus ni moins qu'assimiler la race gauloise, taxée de barbarie. Le grand rêve de César frôle la mégalomanie, ce que lui aussi montre par une certaine promptitude à s'engager dans le combat, fût-ce par infanterie interposée. Les plans des Romains conduisent presque toujours à la perpétration de la violence contre quelque victime. Les légionnaires qui auront bien combattu au cours des affrontements seront récompensés par une visite au cirque, institution romaine qui entérine l'apologie de la violence. Serait-ce à dire que, tout compte fait, les Gaulois réagissent davantage qu'ils n'agissent dans l'entreprise de résistance empreinte de destruction qu'ils mènent ? Examiner les fonctions jouées par la violence dans la série devrait permettre de trouver réponse à cette question.

\section{Pourquoi cette surenchère de violence?}

La bataille représente pour les Gaulois la solution à bien des problèmes. Rien de surprenant à ce que le plan le plus simple, celui qui passe par l'affrontement, soit prisé par ces irréductibles. Dans Astérix gladiateur, le héros déclare : «Voilà 
mon plan : on démolit tout et tout le monde [...] !» Obélix de répondre: "Ça c'est astucieux comme plan!» (Goscinny et Uderzo, 1967b, p. 25) Dans cette perspective, le but n'est pas de terminer le combat au plus vite, mais bien de le vivre, si bien qu'émerge une espèce de mystique de l'affrontement, une poétique du combat. La bataille est dans plusieurs cas esthétisée, les échauffourées prenant presque l'apparence de danses chorégraphiées plutôt que de déchaînements barbares. Dans Astérix en Corse, une théâtralisation rythmique du combat donne à la scène un caractère comique: cette dernière se déroule dans une forêt, mais le cadrage ne permet de voir que la cime des arbres, au-dessus de laquelle phylactères et Romains amochés apparaissent épisodiquement selon un tempo vraisemblablement préétabli (Goscinny et Uderzo, 1973, p. 33). L'affrontement peut aussi avoir des vertus pédagogiques, comme en fait l'expérience Goudurix, à qui son oncle Abraracourcix propose, dans Astérix et les Normands, un programme singulier: il va «apprendr[e] à [s]e battre» (Goscinny et Uderzo, 1967a, p. 17). Mais, surtout, les luttes sont le prétexte, dans tous les albums, à de bonnes parties de rigolade. Dans Astérix et Cléopâtre, Obélix est friand de torture en raison des vertus jubilatoires de celle-ci et non dans l'optique de faire souffrir autrui (Goscinny et Uderzo, 1965b, p. 19). Au nom de la rigolade, les Gaulois n'hésitent pas à tout entreprendre. C'est qu'à leurs yeux, étant donné leur philosophie festive de l'altercation, rien n'a autant de valeur que le rire, instrument violent dans les mains de qui s'en sert avec fourberie : la moquerie altère l'identité de l'autre, comme en fait l'expérience le chef détrôné du village dans Le devin (Goscinny et Uderzo, 1972b, p.15-16). Le rire de supériorité entraîne une inversion des points de repère, qui donne aux 
Gaulois un pouvoir que nul Romain ne possède. De caractère hautement dionysiaque, comme l'a noté Mikhaïl Bakhtine à la suite d'Aristote, le rire confère à l'humain un pouvoir proche de celui de la divinité. "Le rire, don de Dieu, offert uniquement à l'homme, est rapproché du pouvoir de l'homme sur toute la terre, de la raison et de l'esprit qu'il est le seul à posséder. » (Bakhtine, p. 78) Ce rire a un effet cathartique sur le lecteur, qui s'esclaffe en compagnie des Gaulois et voit subitement les tensions accumulées s'amoindrir et disparaître à travers la débâcle d'amusements qui lui sont présentés. Pour Michel Maffesoli,

[c]e qui se manifeste dans le rire, c'est une subversion, qui ne se situe pas comme le contraire de tel ou tel ordre, mais comme l'essai d'un ailleurs - comme utopie. Il s'agit moins de contester un système, et par là même de le conforter, que de s'abstenir. Plutôt que de participer à une logique d'énergie, on a affaire à un ordre de la déperdition, de la dépense. (1984, p. 79)

Les aventures n'ont d'autre but que de conduire au point culminant qu'est le banquet final où chacun, par l'entremise du rire, laisse libre cours à ses pulsions, couronnement de la résistance et du libre arbitre.

Sur le plan purement narratif, la violence joue un autre rôle important: elle constitue l'un des moteurs de l'action. L'élément déclencheur de l'intrigue, dans la bande dessinée Astérix, a souvent fort à voir avec la violence. Qu'il s'agisse d'opposer à l'envahisseur romain des forces susceptibles d'en entraver l'avancée ou de remplir les termes d'un défi reposant sur des prouesses surhumaines, le conflit dynamise invariablement l'action. Dans Le Cadeau de César, la famille de l'aubergiste Orthopédix décide d'élire résidence dans le village des irréductibles en raison de l'accueil violent qui lui a été fait, 
afin de renverser le régime politique en place, en opposant la violence à la violence. Même le climat de paix qui sert d'amorce à pratiquement tous les albums n'est que faux-semblant, car cette paix convie souvent à la bataille et les affrontements attirent les personnages nécessaires pour que l'action avance de perturbation en perturbation. Goscinny et Uderzo ont donc tissé leurs intrigues de manière à conduire le lecteur vers un éclatement de brutalité, mais les manifestations de force conduisent dans pratiquement tous les cas au rire et à la fête : dans Astérix le Gaulois, les villageois ne peuvent que s'esclaffer devant les démonstrations de force physique du Romain Caligula Minus (Goscinny et Uderzo, 1961, p. 18). La violence devient un étalon de mesure, à l'aune duquel s'évaluent les qualités propres à chacun et son utilité au sein de la communauté. Le corps humain, distributeur de baffes à l'envie, peut désormais être considéré comme un outil pourvoyeur de structure, substitut de la technologie : toujours dans Astérix le Gaulois, l'espion romain sursaute devant les prouesses du forgeron qui façonne des armes à mains nues, d'Obélix qui livre ses menhirs à bout de bras et d'Astérix qui transporte seul un char rempli de billots immenses (Goscinny et Uderzo, 1961, p. 15).

Autre fonction de la violence, sûrement la plus importante (car il s'agit du thème récurrent le plus prégnant dans l'ensemble de l'œuvre) : la violence est vecteur d'ordre, mais - et la nuance est capitale - par voie de désordre. Notons d'abord que la civilisation romaine telle qu'elle est présentée dans la série est marquée par un ordre péremptoire qui, pour les peuples non romains, s'avère écrasant, notamment pour les Gaulois jugés au tribunal de Rome dans Les Lauriers de César (Goscinny et Uderzo, 1972a, p. 34), confrontés sur les champs 
de bataille d'Astérix chez les Belges (Goscinny et Uderzo, 1979, p. 43) ou encore violentés par les projets de construction symétriques de César dans Le Domaine des dieux (Goscinny et Uderzo, 1971, p. 4-5). Les Romains ont érigé une société dans laquelle le plus fort impose ses points de repère au détriment du conquis. À l’opposé, dans la position défendue par les héros au nom du droit à la liberté, les lecteurs trouvent leur compte. Pour parvenir à mettre en question l'ordre, les Gaulois ont à leur disposition une arme de choix : le désordre. À l'image d'un défilé de carnaval en mode accéléré, les villageois attaquent les camps romains et en minent les soubassements (voir, par exemple, Goscinny et Uderzo, 1964, p. 10). Qu'il s'agisse d'intervertir le protocole militaire romain dans Astérix le Gaulois (Goscinny et Uderzo, 1961, p. 39), de ridiculiser un athlète exceptionnel en lui prouvant sa petitesse dans Astérix aux jeux Olympiques (Goscinny et Uderzo, 1968, p. 8), de causer un désordre civil ou de transformer une villa romaine en capharnaüm dans Le Domaine des dieux (Goscinny et Uderzo, 1971, p.36 et 45), les Gaulois prisent le renversement de l'ordre. Nicolas Rouvière insiste sur le caractère paradoxal de «la sociabilité des [Gaulois, qui] est agitée et imprévisible[,] faite de banquets, de bagarres et de beuveries » $(2006$, p. 5). La violence dont les villageois sont les pourvoyeurs a pour fonction de déranger l'ordre, fût-il culturel, civil ou militaire, afin de lui substituer un désordre qui se révélera catalyseur d'un nouvel ordre en accord avec la notion de liberté. Comme le prétend Roger Caillois, l'affrontement «traduit la loi de la naissance des nations et correspond aux mouvements viscéraux de nature nécessairement horrible, qui président aux naissances physiques. [...] La guerre brise un ordre paralysé et moribond, elle force l'homme à construire un avenir neuf à 
travers de grandes et d'effroyables ruines. » (1950, p. 231). À ce titre, les représentations de champs de bataille du dessinateur Uderzo rendent compte d'un chaos total (Goscinny et Uderzo, 1970, p. 42). Il semble que dans l'imaginaire gaulois évoqué par nos auteurs, à la violence est accordée la valeur ultime, perçue qu'elle est comme l'équivalent d'un cadeau offert à des visiteurs (Goscinny et Uderzo, 1973, p.11) ou à Obélix lors de son anniversaire (Goscinny et Uderzo, 1976, p. 11), et en tant que telle, elle ne peut qu'avoir un visage positif, d'où un éventuel retour à l'ordre, ce qui laisse pressentir un caractère salutaire à cette débâcle de férocité. Serait-ce à dire que la violence constitue dans certains cas un bienfait?

\section{De l'utilité de la violence}

À vrai dire, pour les Gaulois, la bataille semble relever du jeu, par exemple lorsqu'Astérix prend à partie un soldat romain au son d'une chansonnette qu'il fredonne (Goscinny et Uderzo, 1961, p. 45) ou quand Obélix s'amuse au combat, comme le dit précisément Astérix. Le gros Gaulois est convaincu que ce manège ne peut faire "de mal à personne " (Goscinny et Uderzo, 1964, p. 34), preuve que, dans l'imaginaire gaulois, la violence n'entraîne nulle conséquence fâcheuse. Dans aucun des albums, la guerre que se livrent Gaulois et Romains ne s'avère mortelle pour qui que ce soit, et en cela, elle perd de son sérieux. Même les Romains ont conscience du caractère illusoire de cette opposition, le centurion Gazpachoandalus se prêtant au jeu quand, après avoir participé à un engagement, il dit au héros: «[V]ous ne pouvez plus rien nous faire! Nous sommes déjà massacrés! On vous a bien eus, hein ? (Goscinny et Uderzo, 1973, p.14) La perspective d'une guerre signifie 
pour les Gaulois une fête prochaine, et la représentation graphique des combats ne manque à ce titre pas d'éloquence : ne dirait-on pas que les adversaires, enfants ou adultes, se livrent souvent à une ronde endiablée, semblable à un carrousel, comme si le combat consistait en un amusement? (Goscinny et Uderzo, 1972b, p. 38, 1973, p. 5, et 1976, p. 46) La guerre côtoie de près le jeu; elle procure, d'après Roger Caillois, la volupté dans la destruction : «La jouissance semble la plus grande pour l'homme quand il abîme son semblable. S'il s'y abandonne, elle le laisse parfois pantelant et pâmé. Il l'avoue et s'en vante.» (1950, p. 226) Les manifestations de force conduisent ici au rire, là à la fête. Presque aucune goutte de sang n'est versée dans les albums et si le corps, malmené par les baffes que lui assènent les combattants, se trouve parsemé de bosses, il en devient déformé et non démembré, d'où une reconstruction (bien évidemment caricaturale), d'où aussi un caractère grotesque marqué par l'« exagération, l'hyperbolisme, la profusion, l'excès » (Bakhtine, p. 302), signe que "dans le corps humain, la matière devient créatrice, productrice, appelée à vaincre tout le cosmos, à organiser toute la matière cosmique » (Bakhtine, p. 363). Le corps participe ainsi à un renversement sain qui côtoie l'esprit du carnaval. Obélix, désireux de conserver une trace de ses exploits, ne collectionne pas les scalps, mais bien les casques de soldats romains, récipients vidés de leur contenu, prêts à être remplis de nouveau.

Nous voilà effectivement parvenus sur le terrain du carnaval. Les auteurs ont su choisir un scénario approprié à la réactualisation de la geste du héros - dont François-André Isambert rappelle, à la suite de Henri Hubert, qu'elle est issue du «rituel mimétique de la fête» (p. 154). Les combats présentés dans les albums ne relèvent-ils pas davantage de la 
bastonnade que de l'affrontement en bonne et due forme? L'album Le combat des chefs, par exemple, mise sur des caricatures de luttes afin d'égayer le lecteur, lequel participe à son tour au carnaval, car au cœur de cet événement, les « frontières s'effacent entre le corps et le monde, on assiste à une fusion du monde extérieur et des choses» (Bakhtine, p. 308). Aplusbégalix, le Gaulois costaud, confiant, déterminé, subit une raclée des mains d'Abraracourcix, dont le corps paraît plutôt rabougri, ventru et peu élégant. Au moment du triomphe, le chef s'exclame qu'il est « le plus beau [...], le plus fort [...], le vainqueur ! » (Goscinny et Uderzo, 1966, p. 43), procédant à un détrônement des valeurs instituées défendues par le représentant gallo-romain de César. Selon une logique proche de celle du carnaval, le haut se retrouve en bas et vice versa, dans une débâcle festive. La fête est certes ici à l'honneur et tout prétexte est bon pour se livrer à des inversions carnavalesques, dont la moindre n'est assurément pas celle d'un Astérix, véritable nabot, " exemple-type de l'anti-héros » (Andrieu, p. xii), transformé en un être surpuissant. L'hyperbole confère à la série sa singularité, si appréciée des lecteurs. Dans ce décor, le désordre, contrepoint d'un ordre oppresseur, occupe une place de choix et les inversions, fussent-elles verbales, sonores, physiques ou culturelles, ont leur rôle assigné. Caillois (1950, p. 224) a su noter les ressemblances qu'entretiennent la guerre et la fête, événements distincts pourtant rapprochés dans l'excès qu'ils convoquent. En ce sens, il importe que leurs adversaires offrent une certaine résistance aux Gaulois, de manière que le processus d'inversion s'établisse. Le résultat consiste immanquablement en une bonne dose de rire, générée par voie de violence, et le climat de laisser-aller et de liberté qui ne manque pas d'assaillir le lecteur lui permet, à 
travers un sentiment de participation, de vivre l'aventure aux côtés de ses héros, un rire carnavalesque l'assaillant.

Cette violence n'a rien d'insignifiant. Hautement instituante, elle conduit à l'établissement de points de repère qui, d'album en album, demandent à être réitérés, les adversaires ne les intégrant pas. Chez les Gaulois, la vie est simple. Tout malentendu se règle à coups de violence bien dosée, pour une raison élémentaire : "Que ce soit à l'intérieur d'une nation, ou entre les nations, ce que l'on peut appeler la lutte pour la vie, ou l'affrontement au destin, reste cette composante essentielle du donné social. » (Maffesoli, 1984, p. 12) La violence est inhérente à l'existence humaine; elle est «structurant collectif» (p. 14), intégrée de manières différentes par les Gaulois et les Romains, ce qui les distingue en tant qu'adversaires: si les Romains combattent pour pouvoir éventuellement jouir des fruits de la guerre, pour pouvoir s'amuser après-coup, les Gaulois, eux, s'amusent en se battant, au présent. L'on perçoit dès lors les enjeux de l'antagonisme. Toujours selon Maffesoli : "C'est bien parce que le présent vécu est reconnu comme subversif que le premier objectif du pouvoir est de s'en rendre maître, le découper, l'organiser, le diviser en séquences maîtrisables parce que parcellaires. Ainsi découpé, le temps rentre dans l'ordre de la séparation, devient utile et fonctionnel. »(1984, p.54-55) Les Gaulois vivent les bienfaits d'un présent qui culmine en ce que l'anthropologue nomme un «ordre confusionnel » (1985, p. 14), situation où survient «la perte de l'individualisme et du social dans un confusionnel sociétal indéfini » (p. 17), bien représenté par les débâcles de violence, où la «réversibilité » opère. Est-il surprenant que tous les albums se terminent par un festin visant à célébrer les résultats du combat? Nouvelle apologie à 
peine déguisée de la violence, ces festins ne sont pas loin d'inviter à une frénésie future, la bataille y étant presque chaque fois présente. Bien que, contrairement à bien des bandes dessinées de superhéros par exemple, un péril réel, attesté historiquement, soit ici représenté, la paix est assurée $\mathrm{du}$ fait que, paradoxalement, la violence est définitive dans la série.

La violence servirait-elle en ce sens à combattre la violence? Le barde gaulois oppose à la violence du cirque romain la violence de son chant pour échapper aux lions dans Astérix gladiateur (Goscinny et Uderzo, 1964, p. 41). Faire la guerre pour obtenir la paix ? La lutte a ceci d'instituant qu'elle crée une unité autrement impossible chez les Gaulois, chacun ayant un tempérament si chamailleur qu'il devient difficile d'en arriver à un consensus à propos de quoi que ce soit. Elle crée une masse indifférenciée unie dans son entreprise destructricecréatrice. La violence unit les hommes; elle crée de nouveaux liens de fraternité et d'estime; elle institue "un temps où la société convie tous ses membres à un sursaut collectif qui les place soudain côte à côte, les rassemble, les dresse, les aligne, les rapproche de corps et d'âme [...], une période de forte socialisation, de mise en commun intégrale des instruments, des ressources, des forces» (Caillois, 1950, p. 223). Maffesoli parle, à la suite de Georges Sorel, d'une violence qui est « ce qui rassemble, ce qui brise l'atomisation, ce qui fonde la création collective» $\left(1984\right.$, p. 46) ${ }^{10}$. Goscinny et Uderzo ont mis leurs

\footnotetext{
10 Dans Les jeux et les hommes, Caillois précise à propos des jeux qu'ils «ne trouvent généralement leur plénitude qu'au moment où ils suscitent une résonance complice. [...] La plupart d'entre eux, en effet, apparaissent demande et réponse, défi et riposte, provocation et contagion, effervescence ou tension partagée. » $(1958$, p. 97)
} 
personnages dans des situations où ils goûtent le jeu de la violence, mais ils se sont en même temps amusés eux-mêmes à créer leurs aventures, tout en donnant à l'auditoire un plaisir de lecture incommensurable, d'où, pour suivre la pensée de Frédéric Maguet, un « récit astérixien [qui] dénoue sur le mode burlesque toutes les tensions ressenties dans la réalité quotidienne sur le registre de l'impuissance. Astérix réalise un rêve universel : ne pas subir les contraintes qu'exercent sur les individus des organisations et des pouvoirs qui les dépassent » (p.293). Dans la forme cyclique qu'elle épouse, la bande dessinée Astérix promeut la paix du fait que, paradoxalement, la violence y est définitive. Au début, la paix; à la fin, la paix. L'entre-deux consiste en un long épisode de violence instituante. La vie des Gaulois, je le répète, est simple : entre deux festins, on s'amuse en se chamaillant, peu importe l'identité de l'adversaire. La guerre « inaugure chaque fois une ère nouvelle; un temps s'achève quand elle commence; et quand elle prend fin, commence un autre temps, qui diffère du premier par ses plus visibles qualités» (Caillois, 1950, p. 229). Les ennemis violents sont donc une nécessité, qui permet à l'action de culminer en la création d'un monde neuf.

$\mathrm{Au}$ dire de Maffesoli, «la violence "constructive" [...] est l'expression de la fondation sociale, tout comme [...] la violence "destructive" est la manifestation de l'affirmation individuelle » (1984, p. 25). Dans la série Astérix, le groupe des Gaulois unis autour d'un socle commun cherche à imposer un ordre de réalité différent de celui qu'avalise l'empereur César, lui dont les plans visent chaque fois la destruction de quelque 
communauté au nom d'un ordre imposé par l'institué. Astérix, dans cette perspective, fait profiter ses compagnons de son tempérament "râleur, bagarreur, têtu, colérique » (Andrieu, p. xii), dont les effets atteignent leur plein accomplissement dans l'inversion qui en sous-tend les actions. Astérix demeure un personnage d'exception. Si son physique le désavantage et son statut de célibataire endurci fait sourciller, il n'est que davantage resplendissant dans ses interventions menées à l'emporte-pièce. Les ravages qu'il professe, moyen pour échapper des griffes des Romains ou fin en soi, comportent une dimension caricaturale qui plaît à l'auditoire tout en enseignant à celui-ci les bienfaits d'une violence constructive.

\section{Bibliographie}

ANDRIEU, Olivier. 1999, Le Livre d'Astérix le Gaulois, Paris, Albert René.

BAKHTINE, Mikhaïl. 1970, L'OEuvre de François Rabelais et la culture populaire au Moyen Âge et sous la Renaissance, Paris, Gallimard.

CAILloIs, Roger. 1950, L'Homme et le sacré, Paris, Gallimard.

-. 1958, Les Jeux et les hommes, Paris, Gallimard. 
FREUND, Julien. 1979, «La violence dans ses rapports avec la ville et les communautés », dans Maffesoli, Michel et André Bruston (dir.), Violence et transgression, Paris, Anthropos, p. 35-59.

Goscinny, René et Albert Uderzo. 1961, Astérix le Gaulois, Paris, Dargaud.

-. 1962, La Serpe d'or, Paris, Dargaud.

-. 1963, Astérix et les Goths, Paris, Dargaud.

-. 1964, Astérix gladiateur, Paris, Dargaud.

-. 1965a, Le Tour de Gaule, Paris, Dargaud.

-.1965b, Astérix et Cléopâtre, Paris, Dargaud.

-. 1966, Le Combat des chefs, Paris, Dargaud.

-.1967a, Astérix et les Normands, Paris, Dargaud.

-.1967b, Astérix légionnaire, Paris, Dargaud.

-. 1968, Astérix aux jeux Olympiques, Paris, Dargaud.

-.1969, Astérix et le chaudron, Paris, Dargaud.

-. 1970, La Zizanie, Paris, Dargaud.

-. 1971, Le Domaine des dieux, Paris, Dargaud.

—. 1972a, Les Lauriers de César, Paris, Dargaud.

-. 1972b, Le Devin, Paris, Dargaud.

-. 1973, Astérix en Corse, Paris, Dargaud.

-. 1974, Le Cadeau de César, Paris, Dargaud.

-. 1975, La Grande Traversée, Paris, Dargaud. 
—. 1976, Obélix et compagnie, Paris, Dargaud.

-. 1979, Astérix chez les Belges, Paris, Dargaud.

ISAMBERT, François-André. 1982, Le sens du sacré. Fête et religion populaire, Paris, Minuit.

LASSABLIÈRE, Bernard. Ils sont fous ces humains! Détritus, la bonne conscience d'Astérix. Les intuitions de René Girard chez Goscinny et Uderzo. Paris : L'Harmattan, 2002.

MAFFESOLI, Michel. 1984, Essais sur la violence banale et fondatrice, Paris, Librairie des Méridiens.

-. 1985, L'ombre de Dionysos. Contribution à une sociologie de l'orgie, Paris, Librairie des Méridiens, Klincksiedk.

MAGUET, Frédéric. 1998, « Astérix : un objet d'étude légitime ?». Ethnologie francaise (numéro intitulé « Astérix. Un mythe et ses figures »), $n^{\circ} 3$, t. XXVIII, p. 293-295.

RouviÈre, Nicolas. 2006, Astérix ou les lumières de la civilisation, Paris, Presses Universitaires de France.

-. 2008, Astérix ou la parodie des identités, Paris, Flammarion, coll. «Champs ». 


\title{
Résumé
}

Acculé par les Romains à une résistance apparemment futile, un groupe de Gaulois s'oppose malgré tout à l'avancée de l'envahisseur. Ces rebelles, armés d'une potion les rendant irréductibles, aplatissent littéralement l'adversité au cours d'une histoire qui se répète, à quelques variantes près, d'album en album. Il s'agit ici de se demander si le recours à la violence, auquel sont contraints les Gaulois dans la série Astérix, est nécessaire ou s'il n'apparaît pas, çà et là, quelque peu gratuit. L'observation du rôle joué par la violence dans Astérix permet de déterminer si celle-ci incite à l'agressivité ou si, au contraire, elle ne joue pas plutôt un rôle salutaire, voire dionysiaque. Uderzo et Goscinny, les créateurs de la bande dessinée, semblent avoir mis au point un ingénieux subterfuge leur permettant de distiller les effets d'une violence cathartique auprès de générations de lecteurs.

\begin{abstract}
Forced by the Romans to a likely trivial resistance, a group of Gauls still puts up with the invaders. These rebels, armed with a potion that makes them invincible, literally crush their opponents during a story that repeats itself from comic book to comic book. We will be wondering here if the resort to violence in the comic book series Asterix is really a necessity or if it seems somewhat gratuitous from time to time. The observation of the part played by violence in Asterix brings one to determine if it leads to aggressiveness or if, to the contrary, it plays a beneficial role, maybe even a Dionysian one. Uderzo and Goscinny, the series' creators, seem to have elaborated an ingenuous ploy helping them to disclose a cathartic violence little by little to generations of readers.
\end{abstract}

